\title{
Dynamic simulation of pedestrian behavior in emergency state
}

\author{
Renfen Zeng,Hongwei Gong ,Cuiyun Li,Yinglian Wang,Ruxiong Li
}

Jingdezhen Ceramics Institute, Jiangxi, Jingdezhen, 333000, China

*rfzeng@163.com

Keyword: social force ,self propelled particle,crystalline state;disordered state

Abstract. On the basis of the social force model, we studied various factors which could affect pedestrian escaping from the room on the emergency occurred in a square room (such as fire) .By Simulation the effect of the pedestrian's desired escape can be abtained.The relationship between the average speed and the time of the pedestrian is numerically simulated when the width of the gate is 1 metre.

\section{Introduction}

One of the many disasters of collective action is fleeing of the group in panic,which Often leads to death,such as trampled to death,or crushed to death.Sometimes such behavior is triggered by life-threatening accident ,for example the crowded buildings catch fire; In other cases, Crowded seat is prone to panic,Or there seems to be no reason,Athough engineers are trying to mitigate the extent of the disaster,the frequency of their occurrence seems to increase with the number of group and the size of group,A systematic study of this kind of panic and predicting the quantitative theory of congestion dynamics is still rarely studied.In the Pedestrian behavior model ,panic and crowding mechanism were studied by the unequal movement in the crowd.Through simulation the actual way can be put forward to prevent the enormous pressuren with the congestion ,Shorten the time to run and improve the efficiency of escape.Nowadays, The panic has been mainly studied from the perspective of social psychology.Such a panic is a specific form of collective action that occurs in a state of shortage or a gradual reduction of resources. The individual tends to show a non adaptive and unyielding behavior of the group,such as congestion,Life-threatening crowded, these actions are caused by the spread of the group.Congestion is the result of uncoordinated motion in reality.The literature on social psychology, some media reports and Related videos were studied.According to some practical investigations and some of the seminal data sheets ,the typical features of escape panic are summarized in the following:

(1)People try to go faster than normal.

(2)Starting pushing between individuals and the interaction between people are essentially the interaction between body .

(3)Movement, especially is uncoordinated through the bottleneck.

(4)At the exit, the crowd was found to form an arched plug.

(5)Blocking accumulation

(6))In the crowd, the body's interaction gradually increased, which caused a 
dangerous squeeze, which was enough to make a steel fence bent or knock down a brick wall.

(7)Because of obstacles from falling or injured people, escaping becomes slower.

(8)People have shown the performance of the people, do as others do .

(9)Traditional exports are often overlooked or can not be effectively used in the escape state. These results prompted us to simulate the collective phenomenon of the escape of panic in a multi particle system framework and push the collective phenomenon.The simulation is based on social force in our crowded pedestrian dynamics (social force) [1][2] "especially for describing fatal extrusion.which is observed in a state of panic .

\section{Basic hypotheses and theoretical analysis of the model[3][4][5]}

The social psychological and physical strength of the individual are assumed to affect the individual of the group.Every pedestrian $\mathrm{i}$ of $\mathrm{N}$ pedestrian which has Mass $\mathrm{m}_{\mathrm{i}}$ almost can be along a specific direction at a certain speed $v_{\mathrm{i}}^{0}$.Due to external influence ,pedestrians can not keep the speed of $v_{i}^{0} \vec{e}_{i}^{0}$ in the actual movement process.

At a specific time $\tau_{i}$, the pedestrian will be possible to recover the actual speed under the influence of the thrust;At the same time,his/her velocity change depends on the distance from another pedestrian $\mathrm{j}$ and wall $\mathrm{w}, \vec{f}_{i j}$ and $\vec{f}_{i w}$ can be used to represent the interaction respectively.According to the theory of social force model,the variation of velocity with time is given as follows:

$m_{i} \frac{d v_{i}}{d t}=m_{i} \frac{v_{i}^{0} \vec{e}_{i}^{0}(t)-\vec{v}_{i}(t)}{\tau_{i}}+\sum_{j(\neq i)} \vec{f}_{i j}+\sum_{w} \vec{f}_{i w}$

Perhaps escape in the panic is an unpredictable and dangerous thing, life experiment can not be carried out in real life.The right data can not be found to test the model.The lack of data calls for a more reliable model.Therefore, It can be assumed that the parameters are as follows:Take a general football team as an example,mass $\mathrm{m}=80 \mathrm{~kg}$,desired velocity $v_{i}^{0} \approx 0.6 \mathrm{~m} / \mathrm{s}$, reasonable estimation of relaxation time $\tau_{i}=0.5 \mathrm{~s}$, when $A_{i}=2.0 \times 10^{3} \mathrm{~N}, B_{i}=0.08 \mathrm{~m}$, the distance of maintaining normal desired velocity can be attained by simulation, and it is suitable for the fluid that has been measured through the bottleneck,especially under the condition of $v_{i}^{0} \approx 0.8 \mathrm{~m} / \mathrm{s}$, It is very effective that 1.73 people per second pass through 1

metre-wide gate.parameter $\mathrm{k}=1.2 \times 10^{5} \mathrm{~kg} / \mathrm{s}^{2}$ and $\mathrm{k}=2.4 \times 10^{5} \mathrm{~kg} / \mathrm{ms}$ determine the outcome of the interaction between body.Although in reality,there are some differences between individuals.In order to minimize the number of parameters, the same value is choosed for all the pedestrians . 


\section{Computer simulation and analysis of the results}

Based on hypothesis of the above model, some of the important phenomena of escape in panic wss simulated,And this panic escape was not sensitive to the changes in some of the parameters we had assumed above.If desired velocity was the normal state, the flow of people running out of a room was coordinated., and the regular wass table.But the desired velocity was above $1.5 \mathrm{~m} / \mathrm{s}$, people began to crowd into a group, There is a the irregular arched blocking at the exit. When the arch was broken,the fleeing crowd is like an avalanch,this phenomenon is similar to the observed results.this phenomenon is similar to a particulate matter flowing through a chimney or 1 discontinuous plug found in funnel.However,It's worth noting.blockage of granular material flowing through the chimney or funnel is due to the static friction between particles, and there is no remote interaction. The shift to the blockage has been studied by small exports. To study rather than a change in the driving force In addition to the study of static friction.More important is to study the influence of the change of internal motive force on the behavior of the group.Figure 1 shows the initial state. A pedestrian is standing in a random position in the room of $15 \mathrm{mx} 15 \mathrm{~m}$ (Pedestrian density is 0.7).Figure 2 shows the people in the room initially formed an irregular arch in 10 seconds, if desired velocity is high,at this point in the form of the arch is shown in the figure by simulation. And that's what it means to be "forming a crystalline structure."Figure 3 shows that some people have fled the room in 30 seconds. The people in the room formed an arched structure.Figure 4 shows the relationship between the average speed and time of escape from the room.From the picture we can see, at first because there is no squeeze between each other, everybody was running out of the room, which made the average speed of the group large.If you run out of the room with this speed,so the escape time is very short.However, the constraints of the boundary and the mutual extrusion between pedestrians lead to a higher average speed,which can only be maintained for a very short time.From figure 4,the average speed of the group in less than one second time come down pretty quickly,and then fluctuate between $0-0.5$.This indicates that the blocking state and the traffic state occur intermittently,which is called as a metastable "crystalline state",which affects the passage time.Of course,if desired velocity is higher, a stable "crystalline state" can be formed,and that's just the blockage.

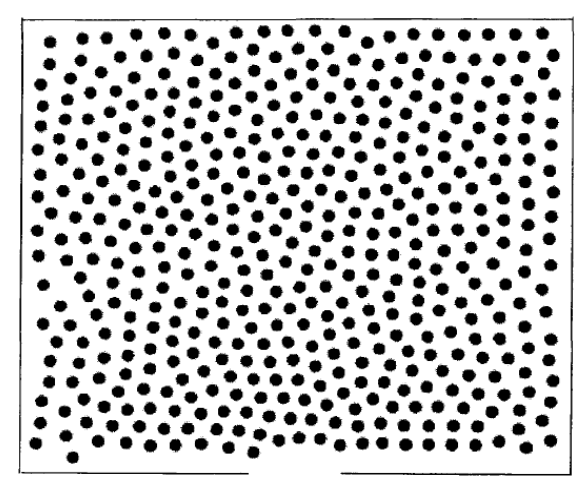

Figure 1.

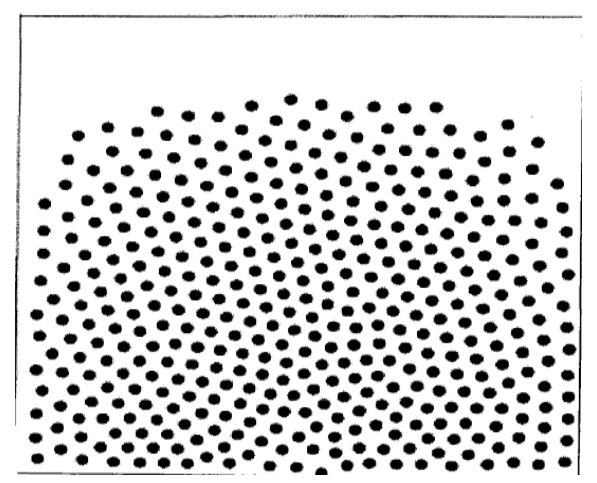

Figure 2. 


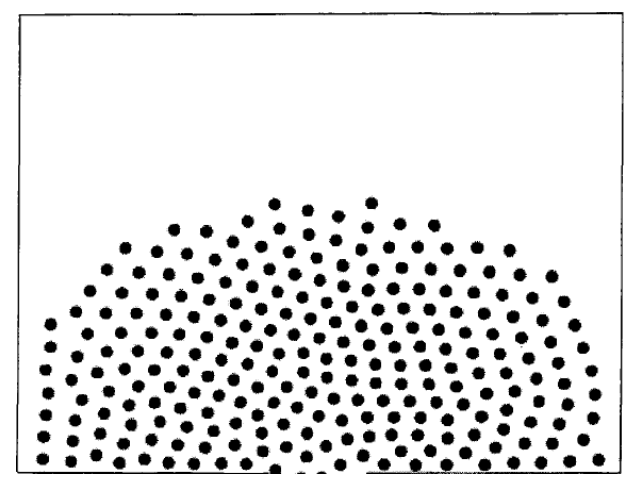

Figure 3.

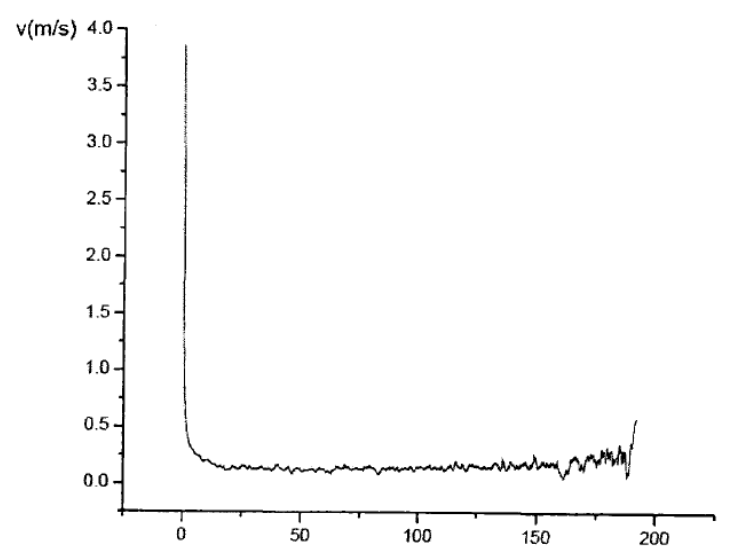

Figure 4.The relationship between average speed and time $(\mathrm{P}=0.7)$ of pedestrian in the 1 metre-wide gate

\section{Summary}

Taking a large building with pedestrian for an example ,the cause of the blockage is analysed .Through the simulation, it is concluded that the speed of the pedestrian escape is faster and easier to plug, which is called "the slower effect". the analysis of this phenomenon make more aware of the deep reason that jam is easy to occur with the increase of the density of traffic flow and the increase of the population density in the building.

\section{References}

[1]D.Helbing,DIPlomthesis,Georg-AngustUniversity,G0ttingen,G-ermany,1990

[2]I.PrigogineandR.Herman,Kinetic Theory of vehieula Traffic(Aweriean Elsevier,NewYOrk,1971)

[3]D.Oeding,Verkehrsbelastung und DimensionierungvonGeh - wegen und anderen AnlagendesFu gaverkehrs(stra $\beta$ enbau und Stra $\beta$ enverkehrstechink,Bonn,1963),vol.22

[4]Highway capacity Manual,Special Report No.209, edited byT ransPortation Research Board National Researeh Coun - cil,Washington,D.C.,1985),ChaP.13. [5]EP.D.NavinandR.J.wheeler,TraffieEng.39,30(1969)Elsevier,NewYOrk,1971). [6]S.J.Cornel et al.,Phys.Rev.Lett.81,1142(1998) 